KeMAS 16 (3) (2021) 317-427
Jirnal Kesehatan Masyarakat

\title{
Liver Function, Leucocyte, and Blood Pressure Levels with Acute Stroke Functional Outcomes
}

\author{
Destika Fahrina ${ }^{1 凶}$, Mahmuddin $^{2}$, Irina Kemala Nasution ${ }^{3}$, Yuneldi Anwar ${ }^{3}$ \\ ${ }^{1}$ Peserta Program Pendidikan Dokter Spesialis Saraf FK Universitas Sumatera Utara/RSUP Haji Adam \\ Malik Medan \\ ${ }^{2}$ Bagian Ilmu Keolahragaan Universitas Negeri Medan \\ ${ }^{3}$ Bagian Neurologi FK Universitas Sumatera Utara/RSUP Haji Adam Malik Medan
}

\section{Article Info}

Article History:

Submitted May 2010

Accepted Augst 2020

Published March 2021

\section{Keywords:}

Heart Function, Leukocyte, Functional Outcome, Stroke, Blood Pressure

\section{DOI}

https://doi.org/10.15294/

kemas.v16i3.24400

\begin{abstract}
Several studies have shown that there is an increase in bilirubin levels, leukocytes, and blood pressure in acute phase strokes. The purpose of this study was to determine the relationship between liver function levels, leucocytes, and blood pressure with the functional outcome of acute stroke. The method was a cross-sectional study with a sample size of 30 people who were selected by consecutive non-random sampling method, clinically proven acute stroke patients, and computed tomography (CT) scan. They were studied at the General Hospital of the Adam Malik Center. Demographic data were analyzed using descriptive statistics. The results showed that all subjects did not differ in terms of demographic characteristics. This study concluds that there is no relation between liver function (total bilirubin, direct bilirubin, indirect bilirubin, serum glutamic oxaloacetic transaminase [AST], serum glutamic pyruvic transaminase [SGPT], alkaline phosphatase [ALP]), leukocytes, and blood pressure with acute stroke functional outcome. The functional outcome in acute hemorrhagic stroke is worse than acute ischemic stroke. Higher levels of liver function, leucocytes, and blood pressure were found in patients with the worse functional outcome though not significantly associated.
\end{abstract}

\section{Introduction}

Stroke is the second most common cause of death after heart disease and the third most common cause of disability worldwide (Kenmogne-domning et al., 2018). Patients after an ischemic stroke, do movement exercises as early as possible to get optimal improvement. Giving movement exercises to patients should involve the family so that it can be done as often as possible. Movement exercises in stroke patients should be done 6 months after a stroke (Mahmudah, 2012).

There is a strong relation between bilirubin levels and the risk of developing ischemic stroke compared to hemorrhagic stroke. Previous studies suggest that this is due to the atherogenic function of bilirubin (Kimm et al., 2009). High glutamate levels and low glutamate oxaloacetate transaminase (GOT) are associated with poor functional outcome on admission. Higher levels of GOT at admission are associated with a good 3-month functional outcome (Ramos-cabrer et al., 2011).

High alkaline phosphatase (ALP) levels are not associated with cerebral atherosclerosis but are a prognostic factor for long-term functional outcomes after ischemic stroke (Kim et al., 2013). Infection such as pneumonia is the most common complication of ischemic stroke. Several studies have shown that systemic inflammation is also a risk factor for ischemic stroke. More specifically, early leukocytosis and neutrophilia are associated with infarct volume in ischemic stroke patients. Increased levels of 
total peripheral leukocytes and neutrophils are also associated with high rates of recurrence in ischemic stroke (Silver, 2013).

Leukocytes interact with platelets, endothelium, and coagulant factors. Acute leukocytosis will shift the homeostatic balance in the presence of coagulation. And play an important role in restraining bleeding after intracerebral hemorrhage (Morotti et al., 2016). Blood pressure in the subacute phase of stroke can affect the 3-month outcome and variability in blood pressure during this period is an independent predictor of clinical outcome (Havenon et al., 2016; Kang et al., 2018).

Blood pressure that is too high or too low in acute ischemic stroke is not beneficial for the improvement of neurological function. Increased blood pressure in patients with acute ischemic stroke at admission is usually associated with a higher NIHSS score. High blood pressure will cause further nervous system damage (Ji et al., 2016). Hypertension is a risk factor for stroke, especially for the incidence and recurrence of intracerebral bleed. It can also be a predictor of poor outcome in the first 24 hours after intracerebral hemorrhage. Increased variability of systolic blood pressure is a predictor of hematoma development and mortality in patients with intracerebral hemorrhage (Manning et al., 2014).

\section{Method}

This research is an analytical descriptive study with a cross-sectional study design from April to September 2019 in stroke patients treated at the Inpatient Care Unit of Rindu A4 Neurology RSUP Haji Adam Malik Medan. The subjects were consecutively taken if they met the inclusion criteria: a) all acute phase stroke patients who were clinically proven to have suffered a stroke and had been confirmed by a CT scan, b) were willing to participate in the study, and there were no exclusion criteria, namely patients with a) recurrent strokes, b) acute stroke in the brain stem, c) stroke with surgical management, d) impaired liver function, e) liver malignancy, f) Sanemia, g) history of alcohol consumption in the last three months, h) history of chronic renal failure, i) history of using non-steroidal antiinflammatory drugs, $\mathrm{j}$ ) a history of the previous infection, k) pneumonia, l) elevated blood sugar levels, $\mathrm{m}$ ) blood malignancies, n) autoimmune diseases.

The collected data was then assessed for its association with the functional outcome of acute stroke based on NIHSS and mRS on day 1 and day 14. Total bilirubin levels in this study were divided into low $(<0.2 \mathrm{mg} / \mathrm{dl})$, normal (0.2-1.0 mg). / dl), high (>1.0 mg / dl) (Harwell, 2013). Direct bilirubin levels are divided into normal (0.0-0.2 mg / dl) and high (>0.2 mg / dl) (Harwell, 2013). Indirect bilirubin levels are divided into low $(<0.2 \mathrm{mg} / \mathrm{dl})$, normal (0.2-0.8 $\mathrm{mg} / \mathrm{dl}$ ) and high (>0.8 mg / dl) (Harwell, 2013). SGOT levels were divided into normal $(<35 \mathrm{U} /$ $\mathrm{L}$ in men) and $<31 \mathrm{U} / \mathrm{L}$ in women) and high ( $\geq$ $35 \mathrm{U} / \mathrm{L}$ in men and $\geq 31 \mathrm{U} / \mathrm{L}$ in women) (W and Brickell, 2007). SGPT levels were divided into normal ( $<45 \mathrm{U} / \mathrm{L}$ in men) and $<34 \mathrm{U} / \mathrm{L}$ in women) and high ( $\geq 45 \mathrm{U} / \mathrm{L}$ in men and $\geq 34$ U / L in women) (W and Brickell, 2007). ALP levels are divided into low $(<53 \mathrm{U} / \mathrm{L}$ in men and $<42 \mathrm{U} / \mathrm{L}$ in women), normal (ALP 53-128 $\mathrm{U} / \mathrm{L}$ in men and 42-98 U / L in women), and high (ALP> $128 \mathrm{U} / \mathrm{L}$ in men and $>98 \mathrm{U} / \mathrm{L}$ in women) (W and Brickell, 2007). Leukocytes were divided into low $(<4,000 / \mu \mathrm{L})$, normal $(4,000-11,000 / \mu \mathrm{L})$ and high $(>11,000 / \mu \mathrm{L})(\mathrm{W}$ and Brickell, 2007). Blood pressure in this study was divided into normal (systole $<120 \mathrm{mmHg}$ and diastole $<80 \mathrm{mmHg}$ ), prehypertension (systole 120-139 $\mathrm{mmHg}$ or diastole 90-99 $\mathrm{mmHg}$ ), stage I hypertension (systole 140-159 $\mathrm{mmHg}$ and diastole 90-99 mmHg. ), and stage II hypertension (systole $\geq 160 \mathrm{mmHg}$ or diastole $\geq 100 \mathrm{mmHg}$ ) (Olin and Pharm, 2018).

\section{Result and Discussion}

Out of 30 acute stroke sufferers, 18 $(60.0 \%)$ suffered from an ischemic stroke when $12(40.0 \%)$ suffered from hemorrhagic stroke. Women suffered ischemic strokes more frequently (53.3\%) than men (46.7). The mean age for ischemic stroke was $60.1 \pm$ 9.8 years, while for hemorrhagic stroke was $57.4 \pm 9.1$ years (Table 1). Hypertension is the most common risk factor for acute stroke (table 1). Based on statistical analysis using the Chi-Square test, it showed that there was no significant relationship between liver function, leucocytes, and blood pressure with 
the functional outcome of acute stroke based on NIHSS and mRS on days 1 (tables 2 and 3 ) and 14 (tables 4 and 5).

The mean age in acute ischemic stroke is higher than for acute hemorrhagic stroke. Women suffer from acute strokes more often than men. However, there was no significant difference in the proportion between acute ischemic stroke and acute hemorrhagic stroke based on gender $(p=0.98)$ (Firoozabadi et al., 2013). The incidence of cerebral infarction and intracerebral hemorrhage was higher in men than in women, although subarachnoid bleeding was higher in women, although this difference was not statistically significant (Appelros, Stegmayr, and Terent, 2009)

Table 1. Demographic Characteristic of Acute Ischemic Stroke and Acute Hemorrhagic Stroke

\begin{tabular}{|c|c|c|c|}
\hline Characteristic & $\begin{array}{l}\text { Acute Ischemic } \\
\text { Stroke }(\mathrm{n}=18)\end{array}$ & $\begin{array}{l}\text { Acute Hemorrhagic Stroke } \\
(\mathrm{n}=12)\end{array}$ & $P$ \\
\hline Age, average \pm SD & $60,1 \pm 9,8$ & $57,4 \pm 9,1$ & $0,332^{\star}$ \\
\hline $\begin{array}{l}\text { Gender, } \mathrm{n}(\%) \\
-\quad \text { Male } \\
\text { - } \quad \text { Female }\end{array}$ & $\begin{array}{l}10(55,6) \\
8(44,4)\end{array}$ & $\begin{array}{l}4(33,3) \\
8(66,7)\end{array}$ & $0,232^{\star \star}$ \\
\hline $\begin{array}{ll}\text { Tribe, } \mathrm{n}(\%) \\
: \quad \text { Batak } \\
\dot{0} \quad \text { Java } \\
\text { Karo } \\
\text { Occupation, } \mathrm{n}(\%)\end{array}$ & $\begin{array}{l}11(61,1) \\
4(22,2) \\
3(16,7) \\
0(0,0)\end{array}$ & $\begin{array}{l}9(75,0) \\
1(8,3) \\
1(8,3) \\
1(8,3)\end{array}$ & $0,577^{* * *}$ \\
\hline $\begin{array}{ll}\text { - } & \text { Farmer } \\
\text { - } & \text { Honorary Employee } \\
\text { - } & \text { State Employee Employee Ret. } \\
\text { - } & \text { Entrepreneur } \\
\text { - } & \text { Housewife }\end{array}$ & $\begin{array}{l}4(22,2) \\
2(11,1) \\
2(11,1) \\
3(16,7) \\
4(22,2) \\
3(16,7)\end{array}$ & $\begin{array}{l}1(8,3) \\
0(0,0) \\
0(0,0) \\
0(0,0) \\
5(41,7) \\
6(50,0)\end{array}$ & $0,011^{\star * *}$ \\
\hline $\begin{array}{ll}\text { Education, } \mathrm{n}(\%) \\
: & \text { Graduate } \\
: & \text { Elementary } \\
\bullet & \text { Junior HS } \\
& \text { Senior HS }\end{array}$ & $\begin{array}{c}4(22,2) \\
0(0,0) \\
2(11,1) \\
12(66,7)\end{array}$ & $\begin{array}{c}0(0,0) \\
1(8,3) \\
1(8,3) \\
10(83,3)\end{array}$ & $0,141^{\star * *}$ \\
\hline $\begin{array}{ll}\text { Risk Factor, } \mathrm{n}(\%) \\
\cdot \quad & \text { Hypertension } \\
\text { - } & \text { Diabetes Mellitus } \\
\text { - } & \text { Heart disease } \\
& \text { Smoking }\end{array}$ & $\begin{array}{l}12(66,7) \\
4(22,2) \\
2(11,1) \\
4(22,2)\end{array}$ & $\begin{array}{c}10(83,3) \\
1(8,3) \\
2(16,7) \\
1(8,3)\end{array}$ & $\begin{array}{l}0,419^{* * * *} \\
0,622^{2 * * *} \\
1,000^{* * * *} \\
0,622^{* * * *}\end{array}$ \\
\hline
\end{tabular}

${ }^{\star} t$-independent test, $p<0,05,{ }^{* \star}$ Uji Chi Square, ${ }^{* * *}$ Mann Whitney test, $\quad p<0,05,{ }^{* * * *}$ Fisher's Exact test, $p<0,05$. Source: Primary Data, 2019

Women have a lower incidence of stroke than men. It may be due to genetic factors and the positive effect of estrogen on the cerebral circulation. However, the stroke risk could double after menopause due to reduced estrogen. Lack of estrogen can lead to the development of cardiovascular disease through changes in the structure and function of blood vessels and affects the coagulation pathway (Appelros, Stegmayr and Terent, 2009; Goldstein et al., 2011).

Hypertension is the most risk factor for stroke in this study. There is a significant relationship between obesity and stress and the incidence of hypertension in women aged 40-55 years. Obesity has the risk of developing hypertension by $\mathrm{OR}=4.2$ times compared to those who are not obese. Stress at work tends to cause severe hypertension. Sources of stress in work include workload, inadequate work facilities, unclear job roles, unclear responsibilities, problems in relationships with other people, and family demands. Health workers should provide health education to the public regarding hypertension on a regular and scheduled basis. Whereas for working women (teachers) are recommended to make lifestyle changes. Namely regular exercise and 
consuming healthy foods so that it can prevent obesity, then for those who are obese it is recommended to reduce body weight so that bodyweight can return to normal, for those who are stressed must be able to control the stress. , avoiding problems that can cause stress and not thinking about things that are not stressful by controlling risk factors associated with hypertension and hypertension can be prevented (Korneliani, 2013)

Table 2. Relation of Liver Function Levels (Total Bilirubin, Direct Bilirubin, Indirect Bilirubin, SGOT, SGPT, ALP), Leukocytes and Blood Pressure with Acute Stroke Severity Based on NIHSS Day 1.

\begin{tabular}{|c|c|c|c|c|c|c|}
\hline & & $\begin{array}{c}\text { Light Outcome } \\
\text { n (\%) }\end{array}$ & $\begin{array}{c}\text { Medium } \\
\text { Outcome } \\
\text { n (\%) }\end{array}$ & $\begin{array}{c}\text { High } \\
\text { Outcome } \\
\text { n (\%) }\end{array}$ & Total & Nilai p \\
\hline $\begin{array}{c}\text { Total } \\
\text { Bilirubin }\end{array}$ & $\begin{array}{l}\text { Low } \\
\text { Normal } \\
\text { High }\end{array}$ & $\begin{array}{l}2(66,7) \\
2(14,3) \\
3(23,1)\end{array}$ & $\begin{array}{l}1(33,3) \\
9(64,3) \\
5(38,5)\end{array}$ & $\begin{array}{c}0(0,0) \\
3(21,4) \\
5(38,5)\end{array}$ & $\begin{array}{c}3(100,0) \\
14(100,0) \\
13(100,0)\end{array}$ & $0,152^{\star}$ \\
\hline $\begin{array}{c}\text { Direct } \\
\text { Bilirubin }\end{array}$ & $\begin{array}{l}\text { Normal } \\
\text { High }\end{array}$ & $\begin{array}{c}0(0,0) \\
4(19,0)\end{array}$ & $\begin{array}{c}9(100,0) \\
10(47,6)\end{array}$ & $\begin{array}{c}0(0,0) \\
7(33,3)\end{array}$ & $\begin{array}{c}9(100,0) \\
21(100,0)\end{array}$ & $0,475^{\star \star}$ \\
\hline $\begin{array}{l}\text { Indirect } \\
\text { Bilirubin }\end{array}$ & $\begin{array}{l}\text { Low } \\
\text { Normal } \\
\text { High }\end{array}$ & $\begin{array}{c}0(0,0) \\
3(15,8) \\
1(12,5)\end{array}$ & $\begin{array}{c}3(100,0) \\
12(63,2) \\
4(50,0)\end{array}$ & $\begin{array}{c}0(0,0) \\
4(21,1) \\
3(37,5)\end{array}$ & $\begin{array}{c}3(100,0) \\
19(100,0) \\
8(100,0)\end{array}$ & $0,358^{\star}$ \\
\hline $\begin{array}{l}\text { Male } \\
\text { SGOT }\end{array}$ & $\begin{array}{l}\text { Normal } \\
\text { High }\end{array}$ & $\begin{array}{c}3(25,0) \\
0(0,0)\end{array}$ & $\begin{array}{l}8(66,7) \\
1(50,0)\end{array}$ & $\begin{array}{c}1(8,3) \\
1(50,0)\end{array}$ & $\begin{array}{l}12(100,0) \\
2(100,0)\end{array}$ & $0,163^{\star *}$ \\
\hline $\begin{array}{c}\text { Female } \\
\text { SGOT }\end{array}$ & $\begin{array}{l}\text { Normal } \\
\text { High }\end{array}$ & $\begin{array}{c}0(0,0) \\
1(14,3)\end{array}$ & $\begin{array}{l}8(88,9) \\
2(28,6)\end{array}$ & $\begin{array}{l}1(11,1) \\
4(57,1)\end{array}$ & $\begin{array}{l}9(100,0) \\
7(100,0)\end{array}$ & $0,193^{\star *}$ \\
\hline $\begin{array}{l}\text { Male } \\
S G P T\end{array}$ & $\begin{array}{l}\text { Normal } \\
\text { High }\end{array}$ & $\begin{array}{c}3(23,1) \\
0(0,0)\end{array}$ & $\begin{array}{c}9(69,2) \\
0(0,0)\end{array}$ & $\begin{array}{c}1(7,7) \\
1(100,0)\end{array}$ & $\begin{array}{c}13(100,0) \\
1(100,0)\end{array}$ & $0,081^{\star *}$ \\
\hline $\begin{array}{c}\text { Female } \\
S G P T\end{array}$ & $\begin{array}{l}\text { Normal } \\
\text { High }\end{array}$ & $\begin{array}{l}1(7,7) \\
0(0,0)\end{array}$ & $\begin{array}{l}8(61,5) \\
2(66,7)\end{array}$ & $\begin{array}{l}4(30,8) \\
1(33,3)\end{array}$ & $\begin{array}{c}13(100,0) \\
3(100,0)\end{array}$ & $0,813^{* *}$ \\
\hline $\begin{array}{l}\text { Male } \\
A L P\end{array}$ & $\begin{array}{l}\text { Low } \\
\text { Normal } \\
\text { High }\end{array}$ & $\begin{array}{c}1(50,0) \\
1(9,1) \\
1(100,0)\end{array}$ & $\begin{array}{c}1(50,0) \\
8(72,7) \\
0(0,0)\end{array}$ & $\begin{array}{c}0(0,0) \\
2(18,2) \\
0(0,0)\end{array}$ & $\begin{array}{c}2(100,0) \\
11(100,0) \\
1(100,0)\end{array}$ & $0,931^{\star}$ \\
\hline $\begin{array}{l}\text { Female } \\
\text { ALP }\end{array}$ & $\begin{array}{l}\text { Low } \\
\text { Normal } \\
\text { High }\end{array}$ & $\begin{array}{l}0(0,0) \\
1(8,3) \\
0(0,0)\end{array}$ & $\begin{array}{c}0(0,0) \\
8(66,7) \\
2(66,7)\end{array}$ & $\begin{array}{l}1(100,0) \\
3(25,0) \\
1(33,3)\end{array}$ & $\begin{array}{c}1(100,0) \\
12(100,0) \\
3(100,0)\end{array}$ & $0,773^{\star}$ \\
\hline Leukocyte & $\begin{array}{l}\text { Normal } \\
\text { High }\end{array}$ & $\begin{array}{l}2(15,4) \\
5(29,4)\end{array}$ & $\begin{array}{l}7(53,8) \\
8(47,1)\end{array}$ & $\begin{array}{l}4(30,8) \\
4(23,5)\end{array}$ & $\begin{array}{l}13(100,0) \\
17(100,0)\end{array}$ & $0,864^{\star}$ \\
\hline $\begin{array}{l}\text { Blood } \\
\text { Pressure }\end{array}$ & $\begin{array}{l}\text { Normal } \\
\text { Pre } \\
\text { Hypertension } \\
\text { Stage } 1 \\
\text { Hypertension } \\
\text { Stage II } \\
\text { Hypertension }\end{array}$ & $\begin{array}{l}1(50,0) \\
1(25,0) \\
1(9,1) \\
1(7,7)\end{array}$ & $\begin{array}{c}0(0,0) \\
2(50,0) \\
8(72,7) \\
9(69,2)\end{array}$ & $\begin{array}{l}1(50,0) \\
1(25,0) \\
2(18,2) \\
3(23,1)\end{array}$ & $\begin{array}{l}2(100,0) \\
4(100,0) \\
11(100,0) \\
13(100,0)\end{array}$ & $0,053^{*}$ \\
\hline
\end{tabular}

${ }^{\star}$ Kruskal-Wallis test, ${ }^{\star \star}$ Mann Whitney test

Source: Primary Data, 2019

Hypertension has a risk of stroke by 4.375 times than those without it. High lipid level is not considered as a risk of stroke (OR 1.375, CI: 0.453 - 4.170). Meanwhile, poor smoking habits cannot be considered a risk of stroke (OR 2.333, CI: $809-6.730$ ). So, it is necessary to control several risk factors for stroke, including hypertension (Sarini, 2007). Stroke in smokers is associated with morbidity. It is related to the fact that cigarette smoke contains $>4000$ different chemicals, including heavy metals and toxins, which trigger the development of free radicals, endothelial dysfunction, and inflammation, causing the atherosclerotic process to continue. Smoking can affect coagulation status. Smoking also causes a reduction in cerebral blood flow, thereby increasing the risk of clot formation and the risk of subsequent strokes through the flow-slowing or static phenomenon (Shah and Cole, 2010).

The greater the amount of nicotine that builds up in the smoker's body, which causes 
addiction and a pleasant taste, so he wants to smoke more. Besides, the intention to quit smoking is high, which is shown by the desire to quit smoking that will determine the success of quitting smoking. Efforts to quit smoking will be in vain if not based on strong intentions. Nevertheless, it is affected by social support factors to stop smoking behavior. If the social environment rejects and is not happy with smoking, the individual will be able to realize his intention stronger. Vice versa, if it is fellow smokers, smokers who plan to quit smoking should inform their social environment, especially the closest people, namely parents and friends, so that they will later support and appreciate the smoker's efforts. However, if the social environment around them does not know, they will smoke in front of them. It will make smokers influenced to continue smoking. And their intention to quit smoking is delayed or not at all. Therefore, the best step for smokers who want to stop their smoking habit is to have the will to quit smoking. Thus, determining the intention to quit smoking can predict the chance of success in quitting smoking (Riska, Linna and Zainal, 2012).

Table 3. Relation of Liver Function Levels (Total Bilirubin, Direct Bilirubin, Indirect Bilirubin, SGOT, SGPT, ALP), Leukocytes and Blood Pressure with Acute Stroke Severity Based on mRS Day 1

\begin{tabular}{|c|c|c|c|c|c|}
\hline & & $\begin{array}{l}\text { Good Outcome } \\
\mathrm{n}(\%)\end{array}$ & $\begin{array}{l}\text { Poor Outcome } \\
\mathrm{n}(\%)\end{array}$ & Total & $p$ Value \\
\hline Total Bilirubin & $\begin{array}{l}\text { Low } \\
\text { Normal } \\
\text { High }\end{array}$ & $\begin{array}{l}3(100,0) \\
5(35,7) \\
6(46,2)\end{array}$ & $\begin{array}{l}0(0,0) \\
9(64,3) \\
7(53,8)\end{array}$ & $\begin{array}{l}3(100,0) \\
14(100,0) \\
13(100,0)\end{array}$ & $0,471^{\star}$ \\
\hline Direct Bilirubin & $\begin{array}{l}\text { Normal } \\
\text { High }\end{array}$ & $\begin{array}{l}6(66,7) \\
8(38,1)\end{array}$ & $\begin{array}{l}3(33,3) \\
13(61,9)\end{array}$ & $\begin{array}{l}9(100,0) \\
21(100,0)\end{array}$ & $0,236^{* *}$ \\
\hline Bilirubin Indirect & $\begin{array}{l}\text { Low } \\
\text { Normal } \\
\text { High }\end{array}$ & $\begin{array}{l}3(100,0) \\
8(42,1) \\
3(37,5)\end{array}$ & $\begin{array}{l}0(0,0) \\
11(59,7) \\
5(62,5)\end{array}$ & $\begin{array}{l}3(100,0) \\
19(100,0) \\
8(100,0)\end{array}$ & $0,180^{*}$ \\
\hline Male SGOT & $\begin{array}{l}\text { Normal } \\
\text { High }\end{array}$ & $\begin{array}{l}7(58,3) \\
1(50,0)\end{array}$ & $\begin{array}{l}5(41,7) \\
1(50,0)\end{array}$ & $\begin{array}{l}12(100,0) \\
2(100,0)\end{array}$ & $1,000^{* *}$ \\
\hline Female SGOT & $\begin{array}{l}\text { Normal } \\
\text { High }\end{array}$ & $\begin{array}{l}4(44,0) \\
2(28,6)\end{array}$ & $\begin{array}{l}5(55,6) \\
5(71,4)\end{array}$ & $\begin{array}{l}9(100,0) \\
7(100,0)\end{array}$ & $0,633^{\star *}$ \\
\hline Male SGPT & $\begin{array}{l}\text { Normal } \\
\text { High }\end{array}$ & $\begin{array}{l}8(61,5) \\
0(0,0)\end{array}$ & $\begin{array}{l}5(38,5) \\
1(100,0)\end{array}$ & $\begin{array}{l}13(100,0) \\
1(100,0)\end{array}$ & $0,429^{* *}$ \\
\hline Female SGPT & $\begin{array}{l}\text { Normal } \\
\text { High }\end{array}$ & $\begin{array}{l}5(38,5) \\
1(33,3)\end{array}$ & $\begin{array}{l}8(61,5) \\
2(66,7)\end{array}$ & $\begin{array}{l}13(100,0) \\
3(100,0)\end{array}$ & $1,000^{* *}$ \\
\hline Male $A L P$ & $\begin{array}{l}\text { Low } \\
\text { Normal } \\
\text { High }\end{array}$ & $\begin{array}{l}2(100,0) \\
5(45,5) \\
1(100,0)\end{array}$ & $\begin{array}{l}0(0,0) \\
6(54,6) \\
0(0,0)\end{array}$ & $\begin{array}{l}2(100,0) \\
11(100,0) \\
1(100,0)\end{array}$ & $0,589^{*}$ \\
\hline Female $A L P$ & $\begin{array}{l}\text { Low } \\
\text { Normal } \\
\text { High }\end{array}$ & $\begin{array}{l}0(0,0) \\
4(33,3) \\
2(66,7)\end{array}$ & $\begin{array}{l}1(100,0) \\
8(66,7) \\
1(33,3)\end{array}$ & $\begin{array}{l}1(100,0) \\
12(100,0) \\
3(100,0)\end{array}$ & $0,197^{\star}$ \\
\hline Leukocyte & $\begin{array}{l}\text { Normal } \\
\text { High }\end{array}$ & $\begin{array}{l}5(38,5) \\
9(52,9)\end{array}$ & $\begin{array}{l}8(61,5) \\
8(47,1)\end{array}$ & $\begin{array}{l}13(100,0) \\
17(100,0)\end{array}$ & $0,431^{\star}$ \\
\hline Blood Pressure & $\begin{array}{l}\text { Normal } \\
\text { Pre } \\
\text { Hypertension } \\
\text { Stage I } \\
\text { Hypertension } \\
\text { Stage II } \\
\text { Hypertension }\end{array}$ & $\begin{array}{l}1(50,0) \\
2(50,0) \\
5(45,5) \\
6(46,2)\end{array}$ & $\begin{array}{l}1(50,0) \\
2(50,0) \\
6(54,5) \\
7(53,8)\end{array}$ & $\begin{array}{l}2(100,0) \\
4(100,0) \\
11(100,0) \\
13(100,0)\end{array}$ & $0,328^{\star}$ \\
\hline
\end{tabular}

${ }^{\star}$ Mann Whitney test, ${ }^{\star \star}$ Fisher Exact test

Source: Primary Data, 2019 
Total bilirubin had no significant relation with stroke severity and functional outcome at baseline ( $\mathrm{p}>0.05)$. The mechanisms underlying this are unclear (Pineda et al., 2009). Circulating total bilirubin levels have a negative relationship with the incidence of ischemic stroke. It may be due to the ability of bilirubin as an anti-oxidant, which triggers low-density lipoprotein (LDL) oxidation. (Zhong, You and Chen, 2017) .

Table 4. Relation of Liver Function Levels (Total Bilirubin, Direct Bilirubin, Indirect Bilirubin, SGOT, SGPT, ALP), Leukocytes and Blood Pressure with Acute Stroke Functional Output Based on NIHSS Day 14.

\begin{tabular}{|c|c|c|c|c|c|c|}
\hline & & $\begin{array}{c}\text { Light } \\
\text { Outcome } \\
\text { n (\%) }\end{array}$ & $\begin{array}{c}\text { Medium } \\
\text { Outcome } \\
\text { n (\%) }\end{array}$ & $\begin{array}{c}\text { High } \\
\text { Outcome } \\
\text { n (\%) }\end{array}$ & Total & p Value \\
\hline $\begin{array}{l}\text { Total } \\
\text { Bilirubin }\end{array}$ & $\begin{array}{l}\text { Low } \\
\text { Normal } \\
\text { High }\end{array}$ & $\begin{array}{l}2(66,7) \\
2(14,3) \\
3(23,1)\end{array}$ & $\begin{array}{l}1(33,3) \\
9(64,3) \\
5(38,5)\end{array}$ & $\begin{array}{l}0(0,0) \\
3(21,4) \\
5(38,5)\end{array}$ & $\begin{array}{l}3(100,0) \\
14(100,0) \\
13(100,0)\end{array}$ & $0,357^{\star}$ \\
\hline $\begin{array}{l}\text { Direct } \\
\text { Bilirubin }\end{array}$ & $\begin{array}{l}\text { Normal } \\
\text { High }\end{array}$ & $\begin{array}{l}3(33,3) \\
4(19,0)\end{array}$ & $\begin{array}{l}6(66,7) \\
9(42,9)\end{array}$ & $\begin{array}{l}0(0,0) \\
7(33,3)\end{array}$ & $\begin{array}{l}9(100,0) \\
21(100,0)\end{array}$ & $0,072^{\star \star}$ \\
\hline $\begin{array}{l}\text { Indirect } \\
\text { Bilirubin }\end{array}$ & $\begin{array}{l}\text { Low } \\
\text { Normal } \\
\text { High }\end{array}$ & $\begin{array}{l}2(66,7) \\
4(21,1) \\
1(12,5)\end{array}$ & $\begin{array}{l}1(33,3) \\
11(57,9) \\
3(37,5)\end{array}$ & $\begin{array}{l}0(0,0) \\
4(21,1) \\
4(50,0)\end{array}$ & $\begin{array}{l}3(100,0) \\
19(100,0) \\
8(100,0)\end{array}$ & $0,209^{*}$ \\
\hline Male SGOT & $\begin{array}{l}\text { Normal } \\
\text { High }\end{array}$ & $\begin{array}{l}5(41,7) \\
1(50,0)\end{array}$ & $\begin{array}{l}5(41,7) \\
0(0,0)\end{array}$ & $\begin{array}{l}2(16,7) \\
1(50,0)\end{array}$ & $\begin{array}{l}12(100,0) \\
2(100,0)\end{array}$ & $0,593^{\star *}$ \\
\hline Female SGOT & $\begin{array}{l}\text { Normal } \\
\text { High }\end{array}$ & $\begin{array}{l}1(11,1) \\
0(0,0)\end{array}$ & $\begin{array}{l}7(77,8) \\
3(42,9)\end{array}$ & $\begin{array}{l}1(11,1) \\
4(57,1)\end{array}$ & $\begin{array}{l}9(100,0) \\
7(100,0)\end{array}$ & $0,157^{\star \star}$ \\
\hline Male $S G P T$ & $\begin{array}{l}\text { Normal } \\
\text { High }\end{array}$ & $\begin{array}{l}6(46,2) \\
0(0,0)\end{array}$ & $\begin{array}{l}5(38,5) \\
0(0,0)\end{array}$ & $\begin{array}{l}2(15,4) \\
1(100,0)\end{array}$ & $\begin{array}{l}13(100,0) \\
1(100,0)\end{array}$ & $0,157^{\star \star}$ \\
\hline Female $S G P T$ & $\begin{array}{l}\text { Normal } \\
\text { High }\end{array}$ & $\begin{array}{l}1(7,7) \\
0(0,0)\end{array}$ & $\begin{array}{l}8(61,5) \\
2(66,7)\end{array}$ & $\begin{array}{l}4(30,8) \\
1(33,3)\end{array}$ & $\begin{array}{l}13(100,0) \\
3(100,0)\end{array}$ & $0,655^{\star \star}$ \\
\hline Male $A L P$ & $\begin{array}{l}\text { Low } \\
\text { Normal } \\
\text { High }\end{array}$ & $\begin{array}{l}1(50,0) \\
4(36,4) \\
1(100,0)\end{array}$ & $\begin{array}{l}1(50,0) \\
4(36,4) \\
0(0,0)\end{array}$ & $\begin{array}{l}0(0,0) \\
3(27,3) \\
0(0,0)\end{array}$ & $\begin{array}{l}2(100,0) \\
11(100,0) \\
1(100,0)\end{array}$ & $0,758^{*}$ \\
\hline Female $A L P$ & $\begin{array}{l}\text { Low } \\
\text { Normal } \\
\text { High }\end{array}$ & $\begin{array}{l}0(0,0) \\
0(0,0) \\
1(33,3)\end{array}$ & $\begin{array}{l}0(0,0) \\
9(75,0) \\
1(33,3)\end{array}$ & $\begin{array}{l}1(100,0) \\
3(25,0) \\
1(33,3)\end{array}$ & $\begin{array}{l}1(100,0) \\
12(100,0) \\
3(100,0)\end{array}$ & $0,170^{*}$ \\
\hline Leukocyte & $\begin{array}{l}\text { Normal } \\
\text { High }\end{array}$ & $\begin{array}{l}2(15,4) \\
5(29,4)\end{array}$ & $\begin{array}{l}7(53,8) \\
8(47,1)\end{array}$ & $\begin{array}{l}4(30,8) \\
4(23,5)\end{array}$ & $\begin{array}{l}13(100,0) \\
17(100,0)\end{array}$ & $0,432^{*}$ \\
\hline $\begin{array}{l}\text { Blood } \\
\text { Pressure }\end{array}$ & $\begin{array}{l}\text { Normal } \\
\text { Pre } \\
\text { Hypertension } \\
\text { Stage I } \\
\text { Hypertension } \\
\text { Stage II } \\
\text { Hypertension }\end{array}$ & $\begin{array}{l}0(50,0) \\
1(25,0) \\
2(18,2) \\
4(30,8)\end{array}$ & $\begin{array}{l}1(50,0) \\
2(50,0) \\
7(63,6) \\
5(38,5)\end{array}$ & $\begin{array}{l}1(50,0) \\
1(25,0) \\
2(18,2) \\
4(30,8)\end{array}$ & $\begin{array}{l}2(100,0) \\
4(100,0) \\
11(100,0) \\
13(100,0)\end{array}$ & $0,053^{* * *}$ \\
\hline
\end{tabular}

Source: Primary Data, 2019

The difference in bilirubin levels between men and women may be due to the presence of estrogen, iron stores in men, and high heme oxidation in men. In stressful situations, sexual hormones in men can cause liver function damage while female sex hormones have a protective effect on liver function (Zhong, You and Chen, 2017). 
Table 5. Relation of Liver Function Levels (Total Bilirubin, Direct Bilirubin, Indirect Bilirubin, SGOT, SGPT, ALP), Leukocytes and Blood Pressure with Acute Stroke Functional Output Based on mRS Day 14.

\begin{tabular}{|c|c|c|c|c|c|}
\hline & & $\begin{array}{c}\text { Good Outcome } \\
\text { n (\%) }\end{array}$ & $\begin{array}{c}\text { Poor Outcome } \\
\text { n (\%) }\end{array}$ & Total & p Value \\
\hline Total Bilirubin & $\begin{array}{l}\text { Low } \\
\text { Normal } \\
\text { High }\end{array}$ & $\begin{array}{l}3(100,0) \\
5(35,7) \\
6(46,2)\end{array}$ & $\begin{array}{l}0(0,0) \\
9(64,3) \\
7(53,8)\end{array}$ & $\begin{array}{l}3(100,0) \\
14(100,0) \\
13(100,0)\end{array}$ & $0,471^{\star}$ \\
\hline Direct Bilirubin & $\begin{array}{l}\text { Normal } \\
\text { High }\end{array}$ & $\begin{array}{l}6(66,7) \\
8(38,1)\end{array}$ & $\begin{array}{l}3(33,3) \\
13(61,9)\end{array}$ & $\begin{array}{l}9(100,0) \\
21(100,0)\end{array}$ & $0,236^{* *}$ \\
\hline Indirect Bilirubin & $\begin{array}{l}\text { Low } \\
\text { Normal } \\
\text { High }\end{array}$ & $\begin{array}{l}3(100,0) \\
8(42,1) \\
3(37,5)\end{array}$ & $\begin{array}{l}0(0,0) \\
11(59,7) \\
5(62,5)\end{array}$ & $\begin{array}{l}3(100,0) \\
19(100,0) \\
8(100,0)\end{array}$ & $0,180^{*}$ \\
\hline Male SGOT & $\begin{array}{l}\text { Normal } \\
\text { High }\end{array}$ & $\begin{array}{l}7(58,3) \\
1(50,0)\end{array}$ & $\begin{array}{l}5(41,7) \\
1(50,0)\end{array}$ & $\begin{array}{l}12(100,0) \\
2(100,0)\end{array}$ & $1,000^{\star *}$ \\
\hline Female SGOT & $\begin{array}{l}\text { Normal } \\
\text { High }\end{array}$ & $\begin{array}{l}4(44,0) \\
2(28,6)\end{array}$ & $\begin{array}{l}5(55,6) \\
5(71,4)\end{array}$ & $\begin{array}{l}9(100,0) \\
7(100,0)\end{array}$ & $0,633^{\star *}$ \\
\hline Male SGPT & $\begin{array}{l}\text { Normal } \\
\text { High }\end{array}$ & $\begin{array}{l}8(61,5) \\
0(0,0)\end{array}$ & $\begin{array}{l}5(38,5) \\
1(100,0)\end{array}$ & $\begin{array}{l}13(100,0) \\
1(100,0)\end{array}$ & $0,429^{* *}$ \\
\hline Female $S G P T$ & $\begin{array}{l}\text { Normal } \\
\text { High }\end{array}$ & $\begin{array}{l}5(38,5) \\
1(33,3)\end{array}$ & $\begin{array}{l}8(61,5) \\
2(66,7)\end{array}$ & $\begin{array}{l}13(100,0) \\
3(100,0)\end{array}$ & $1,000^{\star *}$ \\
\hline Male $A L P$ & $\begin{array}{l}\text { Low } \\
\text { Normal } \\
\text { High }\end{array}$ & $\begin{array}{l}2(100,0) \\
5(45,5) \\
1(100,0)\end{array}$ & $\begin{array}{l}0(0,0) \\
6(54,6) \\
0(0,0)\end{array}$ & $\begin{array}{l}2(100,0) \\
11(100,0) \\
1(100,0)\end{array}$ & $0,589^{*}$ \\
\hline Female $A L P$ & $\begin{array}{l}\text { Low } \\
\text { Normal } \\
\text { High }\end{array}$ & $\begin{array}{l}0(0,0) \\
4(33,3) \\
2(66,7)\end{array}$ & $\begin{array}{l}1(100,0) \\
8(66,7) \\
1(33,3)\end{array}$ & $\begin{array}{l}1(100,0) \\
12(100,0) \\
3(100,0)\end{array}$ & $0,197^{\star}$ \\
\hline Leukocyte & $\begin{array}{l}\text { Normal } \\
\text { High }\end{array}$ & $\begin{array}{l}5(38,5) \\
9(52,9)\end{array}$ & $\begin{array}{l}8(61,5) \\
8(47,1)\end{array}$ & $\begin{array}{l}13(100,0) \\
17(100,0)\end{array}$ & $0,431^{\star * *}$ \\
\hline Blood Pressure & $\begin{array}{l}\text { Normal } \\
\text { Pre } \\
\text { Hypertension } \\
\text { Stage I } \\
\text { Hypertension } \\
\text { Stage II } \\
\text { Hypertension }\end{array}$ & $\begin{array}{l}1(50,0) \\
2(50,0) \\
5(45,5) \\
6(46,2)\end{array}$ & $\begin{array}{l}1(50,0) \\
2(50,0) \\
6(54,5) \\
7(53,8)\end{array}$ & $\begin{array}{l}2(100,0) \\
4(100,0) \\
11(100,0) \\
13(100,0)\end{array}$ & $0,911^{\star}$ \\
\hline
\end{tabular}

Source: Primary Data, 2019

There was no significant relation between direct bilirubin levels and the severity of acute stroke on day one and the functional outcome of acute stroke ( $\mathrm{p}>0.05)$. Direct bilirubin level is not associated with acute stroke severity on admission (Ms et al., 2019). Bilirubin levels were associated with stroke severity at initial hospital admission but not with the functional outcome on discharge. Stroke severity at initial hospital admission may be a mediator of the relationship between functional outcome at hospital discharge and direct bilirubin levels (Pineda et al., 2009).

Direct bilirubin was not associated with stroke severity or functional outcome. However, the results of this study are not in line with previous studies. High bilirubin levels may be associated with oxidative stress and bilirubin may not have an important role in nerve damage (Ushalakshmi and Ramya, 2019). Indirect bilirubin level was not related to acute stroke severity on day one and functional outcome for acute stroke on day 14 ( $p>0.05)$.

This study is not in line with one by Ushalakshmi et al. stated that indirect bilirubin level is associated with stroke severity and the functional outcome of acute stroke upon hospital admission. It may be due to the study of Ushalakshmi et al. The sample size was more and only patients with an onset of 48 hours (Ushalakshmi and Ramya, 2019). Indirect bilirubin is the end product of heme, so it is 
directly proportional to hemoglobin levels. Low levels of indirect bilirubin in acute stroke reflect low hemoglobin levels (Fabbri et al., 2014).

Acute stroke can trigger inflammation in the body. It will disrupt the heme cycle so that there is a reduction in the formation of red blood cells and ultimately reduce the generation of indirect bilirubin (Zhong, You and Chen, 2017). There was no significant relationship between SGOT level in both men and women with acute stroke severity based on NIHSS and $\mathrm{mRS}$ on day 1 and functional outcomes of acute stroke based on NIHSS and MRS on day 14. SGOT levels in men and women were not related to acute stroke severity (Ms et al., 2019).

Serum transaminase was not related to ischemic stroke or subarachnoid hemorrhage. But it was strongly associated with intracerebral hemorrhage. This relation was stronger in men, especially heavy alcohol drinkers and low body mass index. The elevated SGOT levels were associated with body mass index, blood pressure, fasting blood sugar levels, total cholesterol, smoking, and alcohol consumption ( $\mathrm{p}<0.001$ ) (Kim and 2005, 2005).

Liver enzymes are usually associated with several cardiovascular factors. Serum transaminase levels are influenced by alcohol consumption that can increase the risk of intracerebral hemorrhage (Kim and 2005, 2005). Women have lower SGOT levels than men. Estrogen has a protective effect on endothelial cells, but testosterone has the opposite effect (Kim et al., 2013). SGPT levels in men and women with severity and functional outcome of acute stroke based on NIHSS and mRS days 1 and 14. SGPT levels in men and women were not associated with acute stroke severity (Ms et al., 2019).

The incidence of intracerebral hemorrhage and mortality are two times higher in men than women and more frequently in Asian population (Kim et al., 2013). Women have lower SGPT levels than men because women have a lower tendency to drink alcohol and smoke than men (Kim et al., 2013). Biological factors such as estrogen may have a sex difference effect on the incidence of intracerebral hemorrhage and the mortality rate (Kim et al., 2013).

In this study, there was no significant relationship between ALP levels in men and women with acute stroke severity based on NIHSS and mRS on day 1 and functional outcomes based on NIHSS and mRS on day 14 . However, the underlying mechanism is unclear (Ms et al., 2019). Alkaline phosphatase may be associated with recurrence of vascular disease through vascular calcification and is also an early indicator of molecular marker (Ms et al., 2019). Elevated ALP level is associated with cerebral atherosclerosis, where previous studies stated that the prevalence of coronary heart disease and other peripheral arteries was higher in patients who had elevated ALP or phosphate level (Kim et al., 2013).

Patients who have elevated ALP levels have poorer functional outcome after acute ischemic stroke. However, the increase in ALP levels with this functional outcome is unclear (Kim et al., 2013). Elevated ALP can lead to vascular calcification, myocardial fibrosis, and atherosclerosis. Meanwhile, low serum phosphate levels are associated with hypertension and metabolic syndrome or an increased risk of brain infarction in patients undergoing hemodialysis (Zhong, You and Chen, 2017).

Leukocyte level with severity and functional outcome of acute stroke based on NIHSS and mRS on days one and 14. This is in line with the study of Wicaksana et al., 2017 which stated that there was no significant relation between leukocyte levels and functional outcomes based on NIHSS at admission $(\mathrm{p}=1,000)$ and discharge from the hospital ( $\mathrm{p}=0.056)$ (Wicaksana, Adrianto and Rehatta, 2017). One of the factors that influence the severity and tissue damage after stroke is the emergence of acute-phase reactants triggering the acute phase response. Stroke severity based on NIHSS involves several other prognostic factors include acute phase reactants consisted of C-Reactive Protein (CRP), TNF-alpha, IL-6, IL-8, and fibrinogen. C-Reactive Protein has a significant relationship with stroke severity compared to leukocytes (Wicaksana, Adrianto and Rehatta, 2017).

There is no consensus that there is a relation between total leukocyte level and prognosis after acute ischemic stroke. However, 
several studies have shown that total leukocyte level increases in the incidence of acute stroke (Srinivas and $\mathrm{Ha}, 2017)$. Leukocytes play a role in brain parenchymal injury. Including leakage of blood vessels, the release of hydrolytic enzymes, the emergence of free radicals, or the occurrence of thrombosis with clinical manifestations of fever. In this study, the history of previous infection was excluded (Iranmanesh et al., 2014).

The inflammatory and thrombotic components are considered to play a role in increasing levels of leukocytes. There is a hypothesis that markers of inflammation, hypercoagulability, and fibrin breakdown are associated with unstable atherosclerotic conditions that can increase the number of cerebrovascular and cardiovascular events (Iranmanesh et al., 2014). Blood pressure was not significantly related with acute stroke severity and functional outcome according to NIHSS and mRS on days 1 and 14. Patients with both lacunar and non-lacunar strokes had better clinical outcomes with lower NIHSS scores and discharge mRS scores. Blood pressure was not related with functional outcome (Altmann et al., 2015).

Blood pressure at admission is not associated with stroke severity and cannot predict the initial functional outcome in acute stroke survivors. Early neurological improvement occurs at reduced blood pressure, with a mean reduction in systole of about $10 \mathrm{mmHg}$ (Fabbri et al., 2014). The relation between blood pressure and stroke prognosis is not fully explained. Each component of blood pressure affects prognosis (Ji et al., 2016).

The functional outcome of acute stroke is closely related to the type of stroke and initial blood pressure, whereas patients with lacunar strokes and very high blood pressure at admission have a better prognosis (Altmann et al., 2015). High blood pressure is associated with poor short-term outcome. High blood pressure is more related to ventricular system disorders than hematoma volume, thus affecting mortality (Chiquete et al., 2013). Based on research by Bangalore et. al, in 2017, which states that there is a J-shaped / U-shaped relation between systolic blood pressure (below and above $150 \mathrm{mmHg}$ ), diastole (below and above $70 \mathrm{mmHg}$ ), and mean arterial pressure (below and above $100 \mathrm{mmHg}$ ) with death in hospital, not being allowed to go home, and inability to take care of oneself on discharge (Bangalore et al., 2017).

Decreased blood pressure can limit the expansion of the hematoma in patients with intracerebral hemorrhage (Rossi et al., 2011). This study has several limitations. First, things that can affect liver function levels (total bilirubin, direct bilirubin, indirect bilirubin, SGOT, SGPT, ALP), leukocytes and blood pressure were not adjusted. Second, this study only took blood samples for examination of liver function levels and leukocytes during the disease when before the onset of disease were not carried out so that this study could have bias. Third, the sample size is still relatively small to provide representative results.

\section{Conclussion}

There was no significant relation between liver function levels, leukocytes and blood pressure with acute stroke functional outcome based on NIHSS and mRS on day one and 14 .

\section{References}

Altmann, M., Thommessen, B., Ronning, O.M., Reichenbach, A.S., \& Fure, B., 2015. Blood Pressure Differences between Patients with Lacunar and Nonlacunar Infarcts. Brain and Behaviour, 1, pp.1-6.

Appelros, P., Stegmayr, B., \& Terent, A., 2009. Sex Differences in Stroke Epidemiology: A Systematic Review. Stroke, 40(4), pp.10821090.

Bangalore, S., Schwamm, L., Smith, E.E., Hellkamp, A.S., Suter, R.E., Xian, Y., Schulte, P.J., Fonarow, G.C., \& Bhatt, D.L., 2017. Blood Pressure and In-hospital Outcomes in Patients Presenting with Ischaemic Stroke. European Heart Journal, 2017(0), pp.1-9.

Chiquete, E., Guzman, A.O., Sanchez, A.V., Bonnet, J.N., Ramos, M.A.A., Plascencia, P.G., \& Sandoval, J.L.R., 2013. Blood Pressure at Hospital Admission and Outcome After Primary Intracerebral Hemorrhage. Arch Med Sci, 9(1), pp.34-39.

Fabbri, E., Muscari, A., Collini, A., Giovagnoli, M., Napoli, C., Rossi, V., Vizioli, L., Bonfiglioli, A., Magalotti, D., Puddu, G.M., \& Zoli, M., 2014. Changes of Liver Enzymes and Bilirubin During Ischemic Stroke: 
Mechanisms and Possible Significance Changes of Liver Enzymes and Bilirubin During Ischemic Stroke: Mechanisms and Possible Significance. BMC Neurology, 14(1), pp.1-8.

Firoozabadi, M.D., Kazemi, T., Sharifzadeh, G., Dadbeh, S., \& Dehghan, P., 2013. Stroke in Birjand , Iran: A Hospital-Based Study of Acute Stroke. Iranian Red Crescent Medical Journal, 15(3), pp.264-8.

Goldstein, L.B., Bushnell, C.D., Adams, R.J., Appel, L.J., Braun, L.T., Chaturvedi, S., Creager, M.A., Culebras, A., Eckel, R.H., Hart, R.G., Hinchey, J.A., Howard, V.J., Jauch, E.C., Levine, S.R., Meschia, J.F., Moore, W.S., Nixon, J.V.L., \& Pearson, T.A., 2011. AHA / ASA Guideline Guidelines for the Primary Prevention of Stroke A Guideline for Healthcare Professionals From the American Heart Association/ American Stroke Association. American Stroke Association, 42, pp.517-584.

Harwell, G., 2013. Electrolytes. In: Bishop, M.L., Fody, E.P., Schoeff, L.E. Clinical Chemistry: Principles, Techniques, Correlations. Vol.7, pp.346-359. Lippincot William \& Wilkins, Philadelphia.

Havenon, A.D., Bennett, A., Stoddard, G.J., Smith, G., Wang, H., Wold, J., Chung, L., Tirschwell, D.L., \& Majersik, J.J., 2016. Increased Blood Pressure Variability Is Associated with Worse Neurologic Outcome in Acute Anterior Circulation Ischemic Stroke. Hindawi Publishing Corporation, 2016, pp.1-9.

Iranmanesh, F., Sayadi, A., Vakilian, A., \& ZiaSheykholeslami, N., 2014. Relationship between White Blood Cell Count and Mortality in Patients with Acute Ischemic Stroke. Zahedan J Res Med Sci, 2010(3), pp.4-7.

Ji, N., Meng, P., Liu, N., Xu, B., Zhang, G., Zhou, X., \& He, M., 2016. A Reasonable Blood Pressure Level for Good Clinical Outcome After the Acute Phase of Ischemic Stroke. The Journal of linical Hypertension, 18(6), pp.536-542.

Kang, J., Ko, Y., Park, J.H., Kim, W.J., Jang, M.S., Yang, M.H., Lee, J.S., Lee, J., Han, M.K., Gorelick, P.B., \& Bae, H.J., 2018. Effect of Blood Pressure on 3-month Functional Outcome in the Subacute Stage of Ischemic Stroke. American Academy of Neurology, 2012(79), pp.2018-2024.

Kenmogne-Domning, G.H., Kamtchum-Tatuene, J., Noumegni, S.R., Fokoua-Dongmo, C.M., Zafack, J.G., \& Noubiap, J.J., 2018. Cardiac Complications After Stroke: Protocol for a
Systematic Review and Meta-analysis. BMJ Open Access, 2018, pp.8-12.

Kim, H.C., Oh, S.M., Pan, W.H., Ueshima, H., Gu, D., Chuang, S.Y., Fujiyoshi, A., Li, Y., Zhao, L., \& Suh, I., 2013. Association between Alanine Aminotransferase and Intracerebral Hemorrhage in East Asian Populations. Stroke, 72, pp.131-138.

Kim., 2005. Elevated Serum Aminotransferase Level as a Predictor of' Intracerebral Hemmorhage, Stroke, 2005, pp.1642-1648.

Kimm, H., Yun, J.E., Jo, J., \& Jee, S.H., 2009. Low Serum Bilirubin Level as an Independent Predictor of Stroke Incidence. Stroke, 2009 (40), pp.3422-3427.

Korneliani, K., \& D, M., 2013. Obesitas dan Stres dengan Kejadian Hipertensi, Jurnal Kesehatan Masyarakat, 8(2), pp.113-120.

Mahmudah., 2012. Pengaruh Latihan Gerak Terhadap Keseimbangan Pasien Stroke NonHemoragik. Jurnal Kesehatan Masyarakat, 7(2), pp.129-136.

Manning, L., Hirakawa, Y., Arima, H., Wang, X., Chalmers, J., Wang, J., Lindley, R., Heeley, E., Delcourt, C., Neal, B., Lavados, P., Davis, S.M., Tzourio, C., Huang, Y., Stapf, C., Woodward, M., Rothwell, P.M., Robinson, T.G., \& Anderson, C.S., 2014. 13TLN0888 - Anderson Articles SCn Blood Pressure Variability and Outcome After Acute Intracerebral Haemorrhage: A Post-hoc Analysis of INTERACT2, A Randomised Controlled Trial. The Lancet Neurology, 4422(14), pp.1-10.

Morotti, A., Phuah, C.L., Anderson, C.D., Jessel, M.J., Schwab, K., Ayres, A.M., Pezzini, A., Padovani, A., Gurol, M.E., Viswanathan, A., Greenberg, S.M., Goldstein, J.N., \& Rosand, J., 2016. Leucocyte Count and ICH Expansion. Stroke, 47, pp.1-10.

Liu, Y., Liang, X., Xu, X., Dong, M., Jia, S., Lu, C., \& Wei, Y., 2019. Increased Serum Alkaline Phosphatase in Patients with Acute Ischemic Stroke Yang D9X X. Journal of Stroke and Cerebrovascular Diseases, 28(1), pp.21-25.

Olin, B.R., \& Pharm, D., 2018. Hypertension: The Silent Killer: Updated JNC-8 Guideline Recommendations. Alabama Pharmacy Association, 6(1), pp.1-8.

Kim, J., Song, T.J., Song, D., Lee, H.S., Nam, C.M., Nam, H.S., Kim, Y.D., \& Heo, J.H., 2013. 'Brief Report' Serum alkali Phosphatase and Phosphat Cerebral Atherosclerosis and Functional Outcomes After Cerebral Infarction. Stroke. 2013(9), pp.3547-3549.

Pineda, S., Bang, O.Y., Saver, J.L., Starkman, S., 
Yun, S.W., Liesbeskind, D.S., Kim, D., Ali, L.K., Shah, S.H., \& Ovbiagele, B., 2009. Association of Serum Bilirubin with Ischemic Stroke Outcomes. NIH Public Access, 17(3), pp.147-152.

Ramos-cabrer, P., Campos, F., Sobrino, T., Castellanos, M., Blanco, M., RodriguezYanez, M., Serena, J., Leira, R., \& Castillo, J., 2011. High Blood Glutamate Oxaloacetate Transaminase Levels are Associated with Good Functional Outcome in Acute Ischemic Stroke. J Cereb Blood Flow Metab, 31(6), pp.1387-1393.

Riska, R., Linna, S.D., \& Zainal, A., 2012. Penentu Keberhasilan Berhenti Merokok Pada Mahasiswa. Jurnal Kesehatan Masyarakat, 8(1), pp.1-9.

Rossi, P., Mandelli, C., Manganaro, D., Zecca, B., Maestroni, A., Monzani, V., \& Torgano, G., 2011. A Spontaneous Decrease of Blood Pressure Occurs in Acute Ischemic Stroke with Favourable Neurological Course. The Open Neurology Journal, 2011, pp.48-54.

Sarini, O., 2007. Beberapa Faktor Risiko yang Berhubungan dengan Kejadian Stroke (Studi Kasus RSUP Dr.Kariadi Semarang) Tahun 2007. Jurnal Kesehatan Masyarakat, 3(2), pp.153-164.

Shah, R.S., \& Cole, J.W., 2010. Smoking and Stroke: the More You Smoke the More You Stroke.
Expert Review of Cardiovascular Therapy, 8(7), pp.917-932.

Silver, F.L., 2013. White Blood Cell Count is an Independent Predictor of Outcomes After Acute Ischaemic Stroke. European Journal of Neurology, 2013(7), pp.1-9.

Srinivas, K., \& Ha, K., 2017. Prognostic Significance of Total Leucocytes Count in Acute Ischemic Stroke. International Journal of Contemporary Medical Research, 4(4), pp.833-836.

Ushalakshmi, P.S., \& Ramya, A., 2019. A Study on Association between Serum Bilirubin and Acute Ischemic Stroke and Its Prognostic Significance. IOSR Journal of Dental and Medical Sciences, 18(7), pp.64-69.

W, A., \& Brickell, J., 2007. Assessment of Liver Function. Clinical Chemistry, 1, pp.233-266.

Wicaksana, H.Y., Adrianto, Y., \& Rehatta, N.M., 2017. Correlation between White Blood Cell Count and Clinical Severity Based on NIHSS in Acute Ischemic Stroke Patients. Bali Medical Journal, 6(1), pp.130-134.

Zhong, C., You, S., \& Chen, J., 2017. Serum Alkaline Phosphatase, Phosphate, and InHospital Mortality in Acute Serum Alkaline Phosphatase, Phosphate, and In-Hospital Mortality in Acute Ischemic Stroke Patients. Journal of Stroke and Cerebrovascular Diseases. Elsevier Inc., 2017(11), pp.1-11. 